\title{
Thoughts about Improving the Effects of Massage Manipulation Classroom Teaching
}

\author{
Liangbing Yang ${ }^{1}$, Ying Shao ${ }^{2}$, Haikuan Wang ${ }^{3}$ \\ ${ }^{1,2,3}$ Guangzhou University of Chinese Medicine, Guangzhou Guangdong, 510006, China
}

Keyword: Massage Manipulation, Teaching Method, Teaching Effect, Historical Evolution.

\begin{abstract}
The course of massage manipulation has certain specification, which attaches importance to integrate the theory with practice in educational practice. However, students learning the course are usually not those majored in the massage manipulation. Perhaps, they are even not majored in medicine. Therefore, during the process of learning the course of massage manipulation, they usually do not focus on the learning of theoretical knowledge, further to have negative impacts on deeper learning. Therefore, while developing the classroom teaching of massage manipulation, teachers should establish correct guiding ideology, explore more scientific teaching methods to fatherly improve the effects of classroom teaching, so as to offer corresponding guarantees for improving students' learning effects and cultivate the practical abilities of massage manipulation.
\end{abstract}

\section{Introduction}

As one physical therapy, massage manipulation takes Chinese traditional medicine theories as the guideline. Combined with different techniques and substitutions for relevant techniques, it stimulates human body acupoints and specific parts, further to improve the function of human body and cure diseases, which has played an important role in maintaining the health of human and been appreciated by the public. To set up the course of Massage Manipulation-- the interscholastic course of Guangzhou Higher Education Mega Center, and also carry out educational guidance of massage manipulation, teachers not only have to pass on specific theoretical knowledge to students, but also attach importance to practical teaching. Under the guidance of scientific teaching theory, students can master certain massage manipulation, so as to lay a solid foundation for students to obtain better development in future.

\section{Historical Evolution of Massage Manipulation in China}

During the process that ancient Chinese laboring people fought against the disease for a quite long time, they summarized the health-care therapy-massage manipulation. It appeared in Luoyang, Henan for the first time, which made great contributions to the physical health of ancient Chinese people. As an important component of Tuina (Chinese massage), the massage manipulation was firstly put forward in The Inner Canon of Huangdi-the work composed by our ancestors two thousand years ago, as "As those people with insufficient vitality, considering the collateral, carrying out the massage manipulation to improve the situation”. Medical sage Zhang Zhongjing also deeply studied massage manipulation while studying the medicine. Moreover, he also mentioned massage manipulation in Synopsis of Prescriptions of the Golden Chamber [1], as " As those people feel limbs stagnated, carrying out introduction, expiration and inspiration, acupuncture and massage with cream, never blocking nine orifices". After that, relevant medical majors also discussed about the massage manipulation, but there were no great achievements. In Dynasty of Tang, the healthy-care effect of massage manipulation gradually attracted wide attention of the society. Sun Simiao ever mentioned 
the massage manipulation in Thousand Pieces of Gold Formulae for several times, and pointed out that massage manipulation could effectively prevent disease. In Dynasty of Tang, the governmental department even attached importance to the massage manipulation. The prototype of massage manipulation teaching already came into being. A lot of professional medical personnel with massage manipulation skill were cultivated. Accompanied by the activities of economy and trade, it was gradually transmitted to Korea and Japan, which had certain impact on the development of health-care industry in local areas. In Dynasty of Ming, the government paid high attention to massage manipulation and Imperial Academy of Medicine also begun to study the massage manipulation and published a series of works, such as Massage Manipulation for Infants, Pediatrics Massage Manipulation, Infantile Massage. Massage manipulation was promoted to obtain long-term development in this period. In 1980s, Chinese massage manipulation industry was officially formed, which developed greatly and even gradually aroused great attention of international medical circle. Other countries in the world also successively dispatched medical personnel to learn massage manipulation in China, and also invited Chinese massage manipulation technicians to give lectures and implement education in other countries. During the long-term development process, characteristic by the unique effect of maintaining mental and physical health, massage manipulation has been gradually and widely emphasized on by the social public, which has also made certain contributions to promote Chinese health-care career and been gradually and widely recognized by the international society.

\section{Measures of Improving the Classroom Teaching Effects of Massage Manipulation in the New Age}

With the development of massage manipulation industry and its gradually improved importance of in the society, massage manipulation teaching has also begun to attract attentions from relevant departments. Under the guidance of new teaching concept, we try to reform the classroom teaching, with the hope of fatherly improving the teaching quality and promoting students to form good ability of massage manipulation. In the meanwhile of guaranteeing students to obtain good development after graduation, they can also make certain contributions to the modernized construction of Chinese medical and health services. Next, this paper will analyze the measures to improve the classroom teaching effects of massage manipulation, with the hope of actually promoting the teaching quality of massage manipulation to be fatherly strengthened.

\section{Taking Practical Teaching and Skills Training as the Teaching Guideline}

Currently, due to the influence of traditional educational philosophy, Chinese

normal universities and colleges have also been accustomed to applying the model of cultivating technological talents. In the teaching practice, they usually attach too much importance to theoretical teaching. Under the guidance of new teaching concept, some universities and colleges have also begun to explore the practical teaching method. There are usually extreme cases, namely during the process of pursuing practical teaching, theoretical learning is neglected, which seriously restricts the cultivation of students' comprehensive skills and exerts certain bad influence on the continuously healthy development of students in future ${ }^{[2]}$. Based on that, in the teaching of massage manipulation-the interscholastic course of Guangzhou Higher Education Mega Center, teachers should strengthen the practical teaching by combining with the new curriculum reform. On the basis of guaranteeing the quality of theoretical teaching, teachers should gradually take the practical teaching and cultivation of students' massage manipulation skills as the guiding ideology, promote students to reinforce their massage manipulation skills and lay a solid foundation for cultivating students' comprehensive qualities. In the aspect of concrete operation, to give full play to the teaching effect integrated of theory and practice, teachers should also overcome the following problems: emphasizing on teaching but neglecting the learning of students, emphasizing on imparting knowledge but neglecting enlightening students, attaching importance to external teaching form but neglecting the cultivation 
of students' inward enrichment; evaluation is relatively unitary and effect of evaluation cannot be fully played. Students have insufficient understandings about the importance of massage manipulation teaching, whose learning enthusiasm is quite low. Thus they are not willing to devote themselves to practical teaching.

\section{Exploring Flexible Teaching Method, Actually Stimulating Learning Interests of Students}

During the interscholastic course teaching of Guangzhou Higher Education Mega Center, massage manipulation teaching manifests quite obvious practical characteristics, there is relatively quite less theoretical knowledge, which usually plays a certain role of educational guidance. Therefore, classroom teaching mode of ordinary colleges and universities cannot meet the needs of massage manipulation teaching. To guarantee the teaching effects to be fully played, teachers should combine the characteristics of massage manipulation teaching with students' learning abilities and requirements, explore more scientific massage manipulation teaching method, effectively increase the teaching interests and promote students' learning interests to be stimulated to some extent, further to create good conditions for deeply learning massage manipulation. Based on that, while practicing the classroom teaching of massage manipulation, teachers should pay attention to the following contents for fatherly increasing the teaching effects:

First of all, during the process of classroom teaching, teachers should stick to the principle of integrating theory with practical teaching. According to the theoretical teaching contents, teachers should explore corresponding teaching methods, promote classroom teaching contents to be close to the actual life of students and effectively arouse students' learning interests. During the process of organizing concrete classroom teaching activities, teachers should pay attention to communicate with students in time, and also discover problems encountered by students in massage manipulation teaching. Targeted at problems of students, teachers should carry out specific education and guidance. On the basis of solving problems, students can have deeper understandings about relevant problems, further to create conditions for students to reinforce learning effects.

Secondly, teachers should explore more novel and scientific teaching methods. Chinese traditional massage manipulation classroom teaching method is relatively unitary. Interpretation of massage manipulation is not vivid or concrete, thus it is hard for students to form correct understandings about relevant knowledge and skills. The teaching effects are relatively quite poor, which not only affects the learning effects of students, but also has bad influence on cultivating learning interests of students. Therefore, massage manipulation teachers can try to explore more scientific teaching methods and carry out vivid education and guidance over students, further to create conditions for the formation of students' massage manipulation skills [3]. In the aspect of concrete operation, teachers can properly choose situation creation teaching method and offer massage manipulation simulation platform for students; with the help of multimedia teaching method, teachers can strengthen the vividness of teaching; with the help of task-driven teaching method, teachers can gradually lead students to learn deeply, guide them in a scientific way and promote the educational quality to be fatherly improved.

Thirdly, teachers should positively introduce the philosophy of innovative education. Innovative education is also an important educational philosophy in the new era. To promote the teaching effects of massage manipulation to be improved to the high limit, university teachers should also positively introduce the philosophy of innovative education to educational practice, cultivate students' massage manipulation skills and promote their potentials to be stimulated to the high limit, further to lay a solid foundation for the reinforcement of students' massage manipulation skills. Only by introducing the innovative thought to the practical teaching, can students really break through the limitations of traditional teaching and get bigger learning effects in the New Age, so as to consistently strengthen the comprehensive abilities of students.

Finally, teachers should attach more importance to the teaching evaluation, which is also an important link of massage manipulation teaching. In order to actually increase the effects of massage manipulation teaching, teachers should also attach more importance to the teaching evaluation, pay attention to integrate the teachers' evaluation with students' evaluation, integrate the process 
evaluation with result evaluation, integrate the qualitative evaluation with quantitative evaluation, and also combine the dynamic evaluation with static evaluation, let students to form correct understandings about the massage manipulation, offer scientific guidance for the learning of students in the next stage based on the objective evaluation results, promote students' learning effects to be gradually improved and offer corresponding guarantees for students to obtain good achievements.

\section{Paying Attention to Explore Deep Knowledge, Improving the Comprehensive Abilities of Students}

As those students who are not majored in medicine, if they intend to form good massage manipulation skills and obtain better applications in the future society, they should also form good habits of exploration during the process of deepening learning. Under the guidance of teachers, they should gradually make deep explorations of all massage manipulation techniques, further to explore the most suitable massage manipulation technique combined with their own physical qualities, and then increase the effects of massage manipulation. In view of massage manipulation teaching, the key teaching contents are the accuracy and curative effect of massage manipulation. Targeted at certain problems, students must guarantee the persistence of motions, evenness and softness of strength during the process of applying relevant massage manipulation skills, so as to achieve the goal of deepening teaching effects. Massage manipulation skills of students can be widely recognized by the audiences ${ }^{[4]}$. Therefore, targeted at the difficulty of massage manipulation teaching, after teachers complete the basic theoretical interpretation and demonstration of movements for students, they should also organize students to practice with each other, let them feel the degree and comfort of massage manipulation, promote them to form perception of all technological movements and lay a foundation for the actual operation in future, so as to promote students to consistently improve the practical abilities.

\section{Reinforcing Emphasis on Basic Training}

To master basic massage manipulation skills and finally obtain actual effects of guaranteeing their own or others' health, students who are not majored in medicine should possess good thinking ability, proficient massage manipulation techniques and also certain power and strength. During the teaching process of massage manipulation, because of girls' insufficient power and poor durability, it is hard for them to learn persistently. They usually show extremely great interest in learning at the early stage, but they are not willing to insist on learning after a long time, who may eventually give up learning the massage manipulation skills. Therefore, in order to obtain good teaching effects, they should also emphasize on basic training, let students recognize the importance of basic training at the beginning of study and subjectively join in the basic training, so as to promote students to consistently improve their comprehensive qualities and comparatively complete the learning of massage manipulation easily, further to lay a solid foundation for students to obtain good learning effects and strengthen the abilities of massage manipulation ${ }^{[5]}$. Meanwhile, on the basis of reinforce the basic training, students should also learn to protect themselves, so as to avoid non-standard technical movements, which may result in stubby fingers and deformed joints. All these may have bad impacts on mental and physical health of students. Based on that, while passing on relevant massage manipulation technical movements to students, teachers should explain relevant key points of self-protection to students, further to guarantee them to adjust their own joints and muscles. In the meanwhile of protecting their own bodies, students can obtain good learning effects.

\section{Conclusion}

All in all, during the interscholastic course teaching of Guangzhou Higher Education Mega Center, the massage manipulation teaching is a quite important content for students who are not majored in medicine. Moreover, with the increasingly high living standard of people, it has attracted much 
attention from the social public as time goes by. Therefore, to reinforce the effectiveness of classroom teaching in the New Era, massage manipulation university teachers should positively explore diversified teaching methods, carry out scientific educational guidance, promote their practical abilities of massage manipulation to be gradually reinforced, lay a solid foundation for the social life of students in future and also provide certain supports for the stable development of Chinese health-care career.

\section{Acknowledgement}

Foundation Program: Innovative University-strengthening Engineering, Researches based on the Resource Construction of Interscholastic Course of Guangzhou Higher Education Mega CenterMassage Manipulation Researches on Optimized Teaching Mode of Interscholastic Course of Guangzhou Higher Education Mega Center-Massage Manipulation.

\section{References}

[1] Duan Yimei. Several Tries to Improve the Teaching Quality of Massage Manipulation Optional Course, Journal of Dali University, 2014(8):88-90.

[2] Song Xuewu, Lu Yingkui. Effects of Traditional Chinese Massage Manipulation Curing the Physical Pain Sub-health, Seek Medical (second semimonthly), 2013,11(8):165-166.

[3] Luo Shoubin. Routine Operations of Massage Manipulation for Adults, Journal of Frontiers of Medicine, 2013,(13):340-340.

[4] Lei Longming. Improvement of Pushing Manipulation with One-finger Meditation and Teaching Experiences in Chinese Massage Manipulations, Journal of Guangxi University of Chinese Medicine, 2015,18(3):95-96.

[5] Zhang Yisu, Chen Zhiwei. Application of Massage Manipulation to Infantile Health-care and Research Progress, Papers of The 7th Guoxue State Medical Yulu Forum. 2013:363-368. 